\title{
Erosion and abrasion characteristics of high manganese chromium irons
}

\author{
Kishore $^{\mathrm{a}, *}$, P. Sampathkumaran ${ }^{\mathrm{b}}$, S. Seetharamu ${ }^{\mathrm{b}}$ \\ a Department of Metallurgy, Indian Institute of Science, Bangalore 560012, India \\ b Materials Technology Division, Central Power Research Institute, Bangalore 560080, India
}

\begin{abstract}
Chromium irons are well known for their resistance to both oxidation and wear. Additions of manganese to such alloys, as a means to further improve their properties, has attracted the attention of material scientists. This work reports the effects of manganese content and casting section thickness on the mechanical properties and wear of iron alloys containing $17-19 \mathrm{wt} \% \mathrm{Cr}$.

$\mathrm{Fe}-\mathrm{Cr}$ alloys containing 5 and $10 \mathrm{wt} . \%$ manganese are cast in variously-sized gray cast iron moulds. Castings are quenched and tempered. They are then tested for resistance to solid particle impingement erosion and dry-sand rubber wheel abrasion. Increasing the manganese content decreases the hardness and wear resistance. Decreasing the section thickness improves the wear resistance and hardness. Optical microscopy and scanning electron microscopy are used to evaluate the wear properties associated with microstructural constituents.
\end{abstract}

Keywords: Chromium manganese iron; Manganese content; Section size; Heat treatment; Microstructure; Abrasion; Erosion

\section{Introduction}

Improving the wear resistance of material is a continuous challenge in any engineering practice due to the varying mechanisms such as abrasive, erosive or adhesive type [1,2] in operation. Among the bulk wear resistant materials, many are ferrous-based and high chromium iron is one such material with chromium in the range 4-28\%. It contains hard primary carbides $\left(\mathrm{M}_{7} \mathrm{C}_{3}\right)$ in a supporting matrix. Although these carbides are wear resistant, they are brittle. In general, wear resistance is improved by increasing the amount of carbides (by either increasing the carbon content or by microalloying), by resorting to heat treatment or by adopting a suitable cooling rate. The alterations in microstructure through micro-alloying additions in chromium-rich irons have been examined and proved to be beneficial [3-5]. Addition of manganese in chromium-rich irons for further improvement in performance has also attracted the attention of materials scientists. It would increase the matrix toughness characteristics,

\footnotetext{
* Corresponding author. Tel.: +91 80 22932683; fax: +91 8023600472

E-mail addresses: balkis@metalrg.iisc.ernet.in ( Kishore), ssramu@ powersearch.cpri.res.in (P. Sampathkumaran).
}

as manganese is known to be an austenite stabilizer [6]. If, these castings are made in metal moulds, due to higher cooling rate, globular type of fine primary carbides are formed on the surface in contact with the mould material giving rise to an improved wear behaviour [7]. The information on the effect of higher levels of alloying additions (like chromium, manganese, nickel or molybdenum), various types of heat treatment or different cooling strategies, is rather limited. In particular, their impact on structure-property correlations needs to be specially looked into.

In the present work, the effects of manganese addition at two different levels on the three-body abrasion and solid particle erosion wear characteristics of iron chromium alloys have been investigated. In addition, the effect of heat treatment on the wear and hardness properties of the samples cut from the same alloy castings have been investigated.

\section{Experimental}

\subsection{Melting and casting}

The chromium manganese iron was cast in a coreless laboratory induction-melting furnace of capacity $15 \mathrm{~kg}$ and 

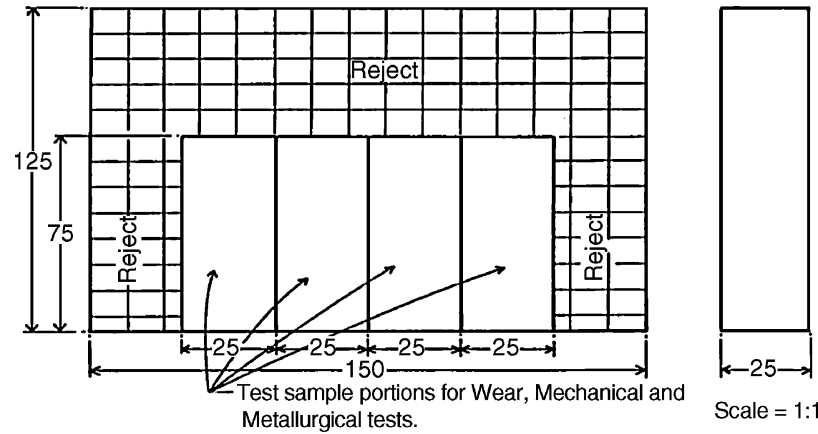

Fig. 1. Dimensions (in $\mathrm{mm}$ ) of metal mould casting.

power rating $15 \mathrm{~kW}$. The permanent (metal) mould made of gray iron, is preheated to $400^{\circ} \mathrm{C}$, before receiving the melt. Charge materials consist of clean mild steel scrap, petroleum coke, ferrochrome, ferromanganese, ferromolybdenum, ferrosilicon and nickel. After melting at about $1400-1450{ }^{\circ} \mathrm{C}$, the charge material is transferred from the furnace into a $700{ }^{\circ} \mathrm{C}$ preheated crucible and subsequently poured into the moulds. The cooled metal castings having the dimensions $150 \mathrm{~mm} \times 125 \mathrm{~mm} \times 12$ or 24 or $40 \mathrm{~mm}$ are stripped from the moulds. The test samples used for wear resistance and hardness measurements, as sectioned from the castings, are of dimensions $75 \mathrm{~mm} \times 25 \mathrm{~mm} \times 6 \mathrm{~mm}$, whereas the metallographic samples are smaller in dimensions measuring $5 \mathrm{~mm} \times 5 \mathrm{~mm} \times 6 \mathrm{~mm}$. Fig. 1 shows the different locations of the test sample portions used for wear, mechanical and metallurgical tests prepared from the castings. Table 1 shows the nominal chemical compositions of the different castings made.

\subsection{Heat treatment}

The test samples are austenitized at $960{ }^{\circ} \mathrm{C}$ for $4 \mathrm{~h}$ in a muffle furnace followed by oil quench to room temperature [8]. Subsequently, they are tempered at $200^{\circ} \mathrm{C}$ for half an hour followed by air-cooling to ambient conditions.

\subsection{Characterization}

The structure-property correlations of the high chromium manganese irons are carried out both in the as-cast (AC) and heat treated (HT) conditions. The microstructural features are characterized using optical microscope and scanning electron microscope (SEM). The three-body abrasion resistance and solid particle erosion resistance properties are evaluated using rubber wheel abrasion test and jet erosion test facilities, respectively. Hardness parameter is measured using a Rockwell hardness tester. XRD stress analyzer equipment with chromium as the target material was used for the estimation of the percentage of retained austenite (RA). Further, the verification of RA was done using NBS standard with $15 \%$ austenite in ferrite and other secondary standards. The carbide volume percentage (CV) of chromium manganese iron samples is determined using an image analyzer attached to the optical microscope. The results of hardness, RA and CV measurements are shown in Table 2.

\subsubsection{Rubber wheel abrasion test}

The rubber wheel abrasion test details are described elsewhere [9]. Briefly stated, the procedure consists of the abrasive silica sand (AFS 60 grade) being fed at the interface between the rotating rubber wheel $(200 \mathrm{rpm})$ and the test sam-

Table 1

Nominal composition of manganese chromium irons

\begin{tabular}{|c|c|c|c|c|c|c|c|c|c|c|}
\hline \multirow[t]{2}{*}{ Serial number } & \multirow[t]{2}{*}{ Casting designation } & \multirow[t]{2}{*}{ Section thickness (mm) } & \multicolumn{8}{|c|}{ Composition, wt.\% } \\
\hline & & & $\mathrm{C}$ & $\mathrm{Mn}$ & $\mathrm{Cr}$ & $\mathrm{Ni}$ & Mo & $\mathrm{Si}$ & $\mathrm{P}$ & $\mathrm{S}$ \\
\hline 1 & $5 \mathrm{M} 12$ & 12 & 2.36 & 4.5 & 18.58 & 0.91 & 1.87 & 2.69 & 0.080 & 0.046 \\
\hline 2 & $5 \mathrm{M} 24$ & 24 & 2.39 & 4.6 & 18.62 & 0.92 & 1.82 & 2.78 & 0.090 & 0.044 \\
\hline 3 & $5 \mathrm{M} 40$ & 40 & 2.55 & 4.8 & 17.90 & 0.81 & 1.90 & 2.10 & 0.075 & 0.057 \\
\hline 4 & $10 \mathrm{M} 12$ & 12 & 2.32 & 9.7 & 17.65 & 0.80 & 1.85 & 1.90 & 0.090 & 0.040 \\
\hline 5 & $10 \mathrm{M} 24$ & 24 & 2.35 & 9.7 & 17.60 & 0.82 & 1.80 & 1.85 & 0.085 & 0.040 \\
\hline
\end{tabular}

Table 2

Summary of hardness, retained austenite percent and carbide volume percent measurements on manganese chromium iron samples

\begin{tabular}{|c|c|c|c|c|c|c|}
\hline \multirow[t]{2}{*}{ Serial number } & \multirow[t]{2}{*}{ Casting designation } & \multirow[t]{2}{*}{ Hardness (HRC) } & \multicolumn{2}{|l|}{ RA $\%$} & \multicolumn{2}{|l|}{$\mathrm{CV} \%$} \\
\hline & & & Range & Average & Range & Average \\
\hline \multirow[t]{2}{*}{1} & $5 \mathrm{M} 12 \mathrm{AC}$ & 56 & $52-55$ & 54 & $27-30$ & 29 \\
\hline & 5M12 HT & 63 & $45-48$ & 47 & & \\
\hline \multirow[t]{2}{*}{2} & $5 \mathrm{M} 24 \mathrm{AC}$ & 55 & $56-58$ & 57 & $26-29$ & 27.5 \\
\hline & $5 \mathrm{M} 24 \mathrm{HT}$ & 61 & $49-51$ & 50 & & \\
\hline \multirow[t]{2}{*}{3} & $5 \mathrm{M} 40 \mathrm{AC}$ & 52 & $59-62$ & 61 & $25-28$ & 26.5 \\
\hline & $5 \mathrm{M} 40 \mathrm{HT}$ & 56 & $55-59$ & 57 & & \\
\hline \multirow[t]{2}{*}{4} & $10 \mathrm{M} 12 \mathrm{AC}$ & 52 & $58-62$ & 60 & $24-28$ & 26 \\
\hline & 10M12 HT & 55 & $52-57$ & 55 & & \\
\hline \multirow[t]{2}{*}{5} & $10 \mathrm{M} 24 \mathrm{AC}$ & 50 & $61-65$ & 63 & $24-27$ & 25.5 \\
\hline & 10M24 HT & 53 & $59-61$ & 60 & & \\
\hline
\end{tabular}


ple, whose initial weight is measured using a high precision electronic balance. The sample is then dead weight loaded $(13 \mathrm{~kg})$ against the rotating wheel for a fixed number of revolutions. At the end of 6000 revolutions, the sample is cleaned, dried and weighed again in the same balance for noting its final weight. The difference in the initial and final weight readings of the sample gives the abrasion wear loss.

\subsubsection{Solid particle erosion test}

The technical details of this are described elsewhere [9]. The procedure consists of the abrasive particles, carried by a stream of compressed air, being made to impinge through a ceramic nozzle of diameter $5 \mathrm{~mm}$ on the test sample, whose initial weight is noted using a digital electronic balance. During the run, the chamber containing abrasive particles gets pressurized and operates the ball valve resulting in particle flow through the jet nozzle at high velocity. The velocity of the particle jet flow as well as the mass flux rate are controlled using the pressure and abrasive feed control valves, respectively. At the end of the test, the sample is cleaned and weighed again in the same balance. The erosion wear loss is the weight difference between the initial and final weights of the sample. The erosion experiments are carried out using silica sand (AFS 60 grade) as the abrasive medium at a particle velocity of $35 \pm 2 \mathrm{~m} / \mathrm{s}$. A mass flow rate in the range $600-700 \mathrm{~g} / \mathrm{min}$ is employed for the time duration of $30 \pm 2 \mathrm{~s}$. The angle of impact in this work is maintained at $45^{\circ} \pm 2^{\circ}$. The particle velocity measurement is measured using a double disc device [10], which is based on the time of flight principle.

In all the abrasion and erosion experiments as well as hardness recordings, the reported value is the average of three measurements on two representative samples. The repeatability of wear tests is ascertained by determining the coefficient of variation [11], which is well with in the acceptable limit of $20 \%$, in all these data sets. The volume loss values used in different plots presented here are calculated from abrasion and erosion weight measurements.

\section{Results and discussion}

Figs. 2 and 3 show the optical micrographs corresponding to samples 5M24 and 10M24, respectively. The first sam-

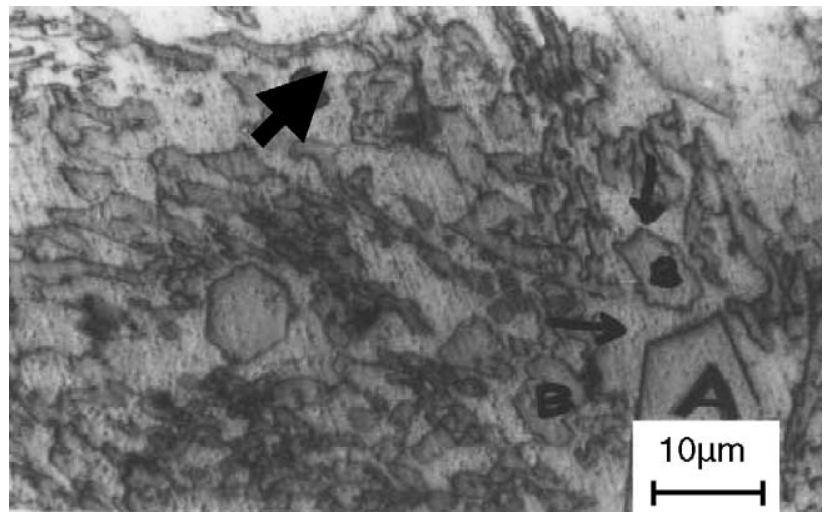

Fig. 2. Optical micrograph of as-cast 5M24 sample.

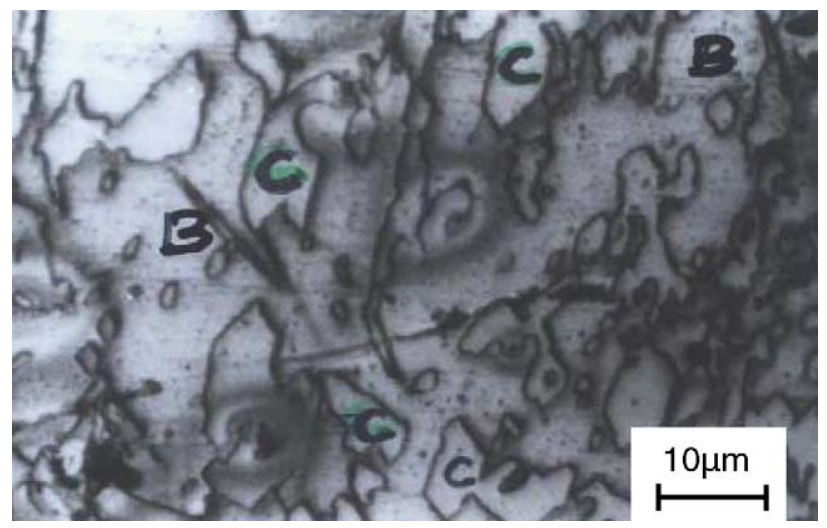

Fig. 3. Optical micrograph of as-cast 10M24 sample.

ple 5M24 shows higher hardness (Table 2) due to the presence of primary $\left(\mathrm{M}_{7} \mathrm{C}_{3}\right)$ carbides of medium-size (marked 'B') and occasional hexagonal (marked 'A') types (Fig. 2) in an austenitic matrix (indicated by arrows), whose content was much lower (i.e., 57\%; Table 2). On the other hand, the sample 10M24 shows coarser primary carbides (marked ' $\mathrm{C}$ ' in Fig. 3) with higher austenite content (marked 'B': 63\%; Table 2). Also, CV for the sample 5M24 is slightly higher (27.5\%) than that noticed for 10M24 (25.5\%) case. The abrasion and erosion data for these samples are shown in Fig. 4a and $b$, respectively. It is seen from these figures that the $10 \%$ manganese sample shows higher abrasion (Fig. 4a) and erosion (Fig. 4b) losses compared to the 5\% case. Thus, there is
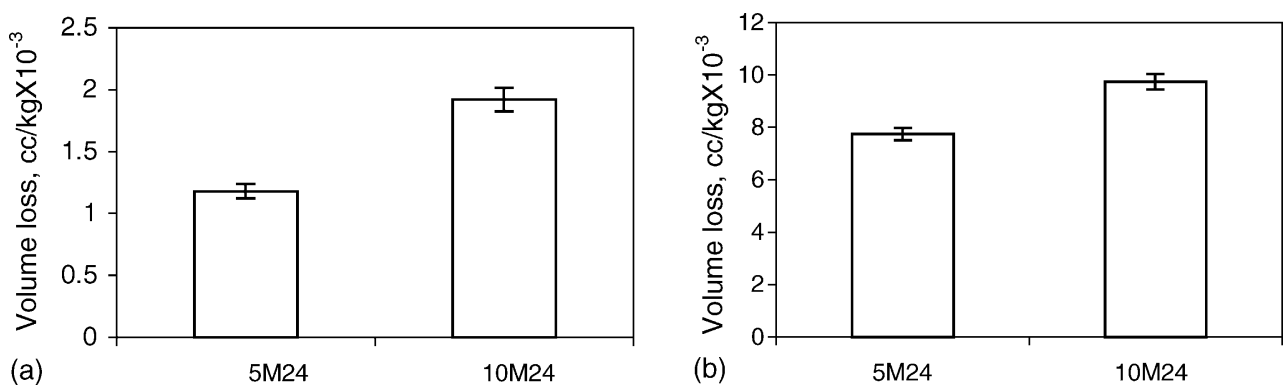

Fig. 4. (a) Loss due to abrasion in 5 and $10 \%$ manganese bearing metal cooled as-cast samples (b) loss due to erosion in 5 and $10 \%$ manganese bearing metal cooled as-cast samples. 
a consistency in the trends irrespective of the test parameter evaluated, whether abrasion or erosion. It is well known that higher the grain or the carbide size [12], lower is the strength and/or hardness and hence lower the wear resistance. This is consistent with lowering of the hardness and wear resistance in $10 \%$ manganese system due to larger size carbides and higher austenite retention in the matrix. Thus, a good correlation amongst the abrasion, erosion and hardness data with the microstructure has been established for the manganese content increase (i.e., from 5 to $10 \%$ ) in such systems.

It is seen from the published literature [13], that effecting a change in manganese content from 0.5 to $4.5 \%$ in a 11-12 wt.\% chromium iron in one case and increasing the manganese content from 1.6 to $3.1 \mathrm{wt} . \%$ in high chromium $(17.5 \%)$ iron, in the other, record a decrease in wear resistance of these alloy systems. In the present case, the lower manganese bearing sample (5M24) shows improved wear resistance compared to the higher manganese bearing counterpart. Thus, the data trends from the literature [13] and the present findings are in good agreement with each other. The microstructural aspects influencing both the fracture toughness and abrasion resistance in white cast irons, emphasized in a published work [14], are corroborated in this work. It is reported that the $\mathrm{CV} \%$ in high chromium iron influences the abrasion volume loss for both the austenitic and the martensitic irons. Further, when the carbide volume is more, both abrasion resistance and hardness values increase. The experimental results on metal cast samples viz. higher abrasion resistance achieved for the sample having higher carbide volume in chromium iron systems, reported in the present work, is in agreement with that reported in the literature [14].

For studying the effect of section size on the wear properties, besides the microstructural features presented earlier for 5M24 (Fig. 2), the optical micrographs for 5M12 and 5M40 are additionally presented in Figs. 5 and 6, respectively. The abrasion and erosion data for samples 5M12 and 5M40 are shown in Fig. 7a and b, respectively along with the data for $5 \mathrm{M} 24$ presented earlier (Fig. 4a and b). It is observed that as the section size decreases (with an associated increase in the cooling rate), the wear losses also decrease. The lower abrasion and erosion losses in case of 5M12 compared to 5M24 and 5M40 is mainly due to: (a) an increase in CV from 26.5 to $29 \%$, (b) an increase in hardness level from 52 to 56 HRC and (c) a decrease in RA from 61 to 54\% (refer Table 2). The

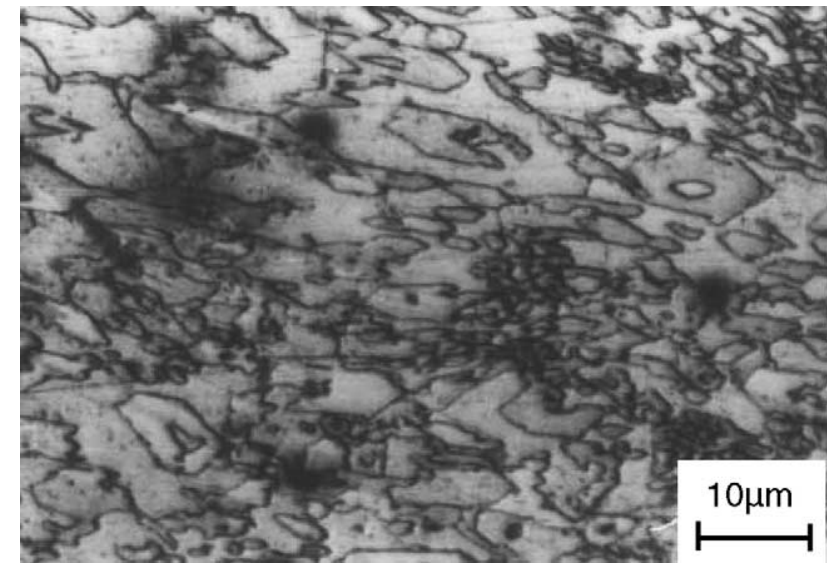

Fig. 5. Optical micrograph of as-cast 5M12 sample.

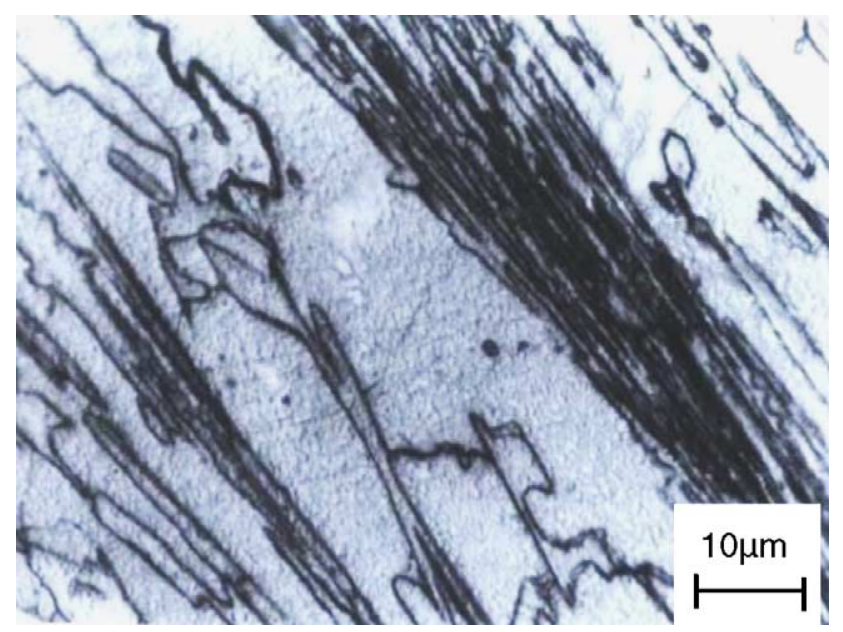

Fig. 6. Optical micrograph of as-cast 5M40 sample.

microstructure depicting fine plus hexagonal primary carbides in the matrix for the 5M12 sample (Fig. 5) supports the trend. Increasing the casting section size to $24 \mathrm{~mm}$ results in formation of primary $\left(\mathrm{M}_{7} \mathrm{C}_{3}\right)$ medium-sized and occasional hexagonal carbides as seen in Fig. 2. A further increase in the section thickness (to $40 \mathrm{~mm}$ ) yields massive (Fig. 6) primary carbides, which are much larger in size compared to 12 and $24 \mathrm{~mm}$ section-sized samples. Thus, a correlation has been established amongst casting section size, wear property data, hardness and the microstructure for the $5 \%$ manganese case.
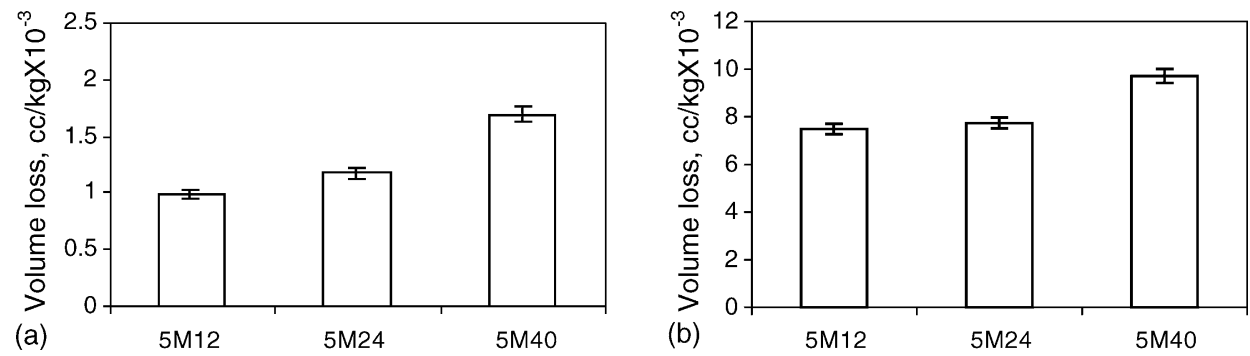

Fig. 7. (a) 5\% metal cooled as-cast samples of different section sizes showing response to abrasion (b) $5 \%$ metal cooled as-cast samples of different section sizes showing response to erosion. 


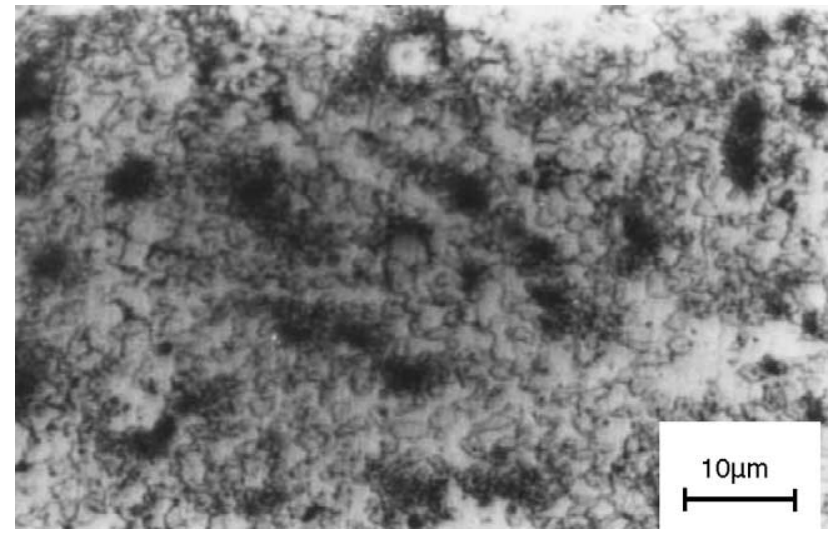

Fig. 8. Optical micrograph of as-cast 10M12 sample.

The optical microscopic features for the $10 \%$ manganese bearing sample are presented in Fig. 8. The abrasion and erosion data for samples 10M12 and 10M24 are shown in Fig. 9a and $b$, respectively. The trend in this case is similar to that for $5 \%$ case discussed earlier. Microstructure wise $10 \mathrm{M} 12$ shows fine primary carbides (Fig. 8) in an austenitic (60\%; Table 2) matrix and higher hardness (52 HRC) compared to 10M24 (Fig. 3). The latter, on the other hand, displays primary carbides, which are coarser compared to 10M12 with slight increase in the austenite $(63 \%)$ content. Therefore, the lower section size sample (10M12), due to the presence of finer carbide morphological features, records lower wear losses (i.e., higher wear resistance). Thus, good correlation amongst tribological (wear) parameters, hardness and microstructure in this $10 \%$ case too could be established in this work.

In an earlier work carried out on high chromium iron (with low manganese) [7], it is seen that the erosion resistance of faster cooled samples is better compared to the slower cooled ones. Further, the erosion resistance is higher at the surface and the sub-surface due to the chilling effect produced due to the use of the metal mould. The erosion resistance decreases towards the core of the cast material. It is inferred that the difference in the erosion resistance at the core and at the surface is due to the appearance of globular type of fine carbides near the surface. In the present work on manganese containing chromium irons, one observes a similar trend of higher wear resistance in the lowest section $(12 \mathrm{~mm})$ size sample

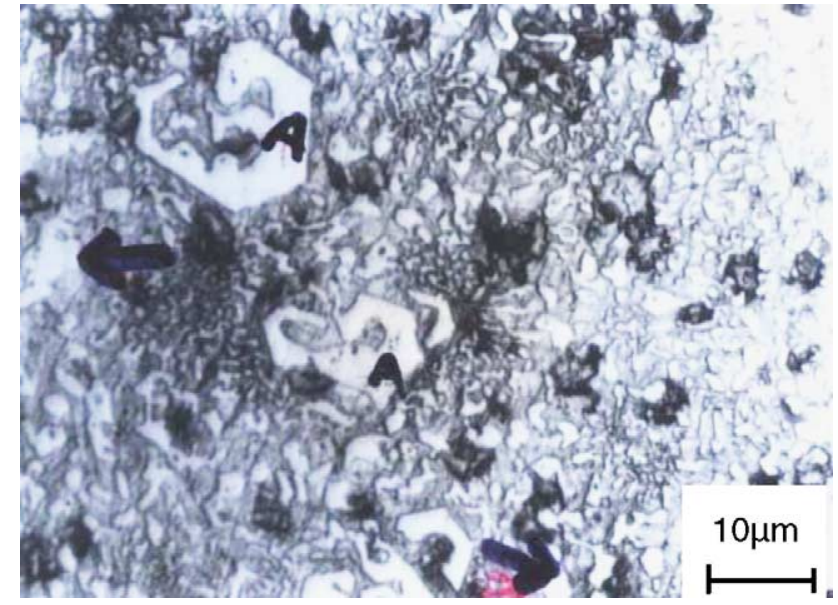

Fig. 10. Optical micrograph of heat treated 5M24 sample.

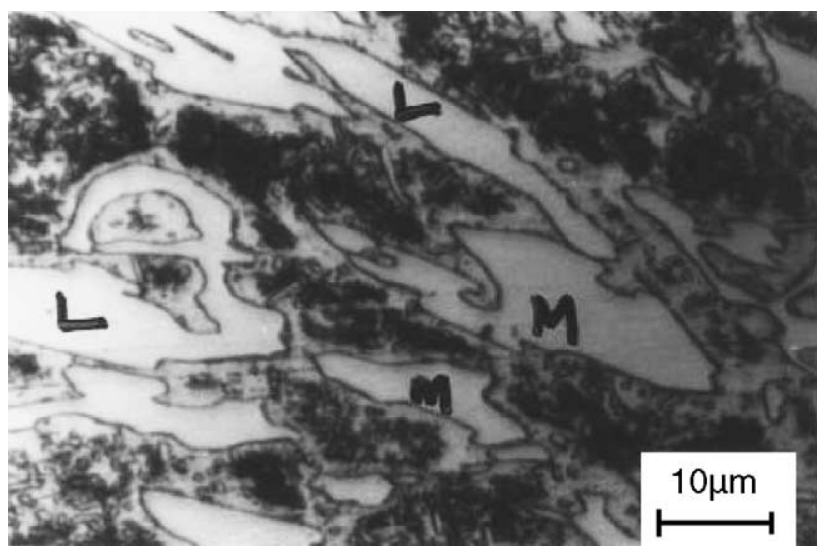

Fig. 11. Optical micrograph of heat treated 10M24 sample.

compared to the $40 \mathrm{~mm}$ section size sample. The reason for such a trend is clearly attributed to the higher cooling rate prevailing in the former case.

It is worth comparing the present data with another work [15] wherein a chilling arrangement on one side of the casting is used. The samples drawn from the chilled side as well as that away from the chilled zone are subjected to abrasion test. The results show that the sample prepared from the chilled area (having faster cooling rate as in lower section size in the present work) yields a higher abrasion resistance. At this
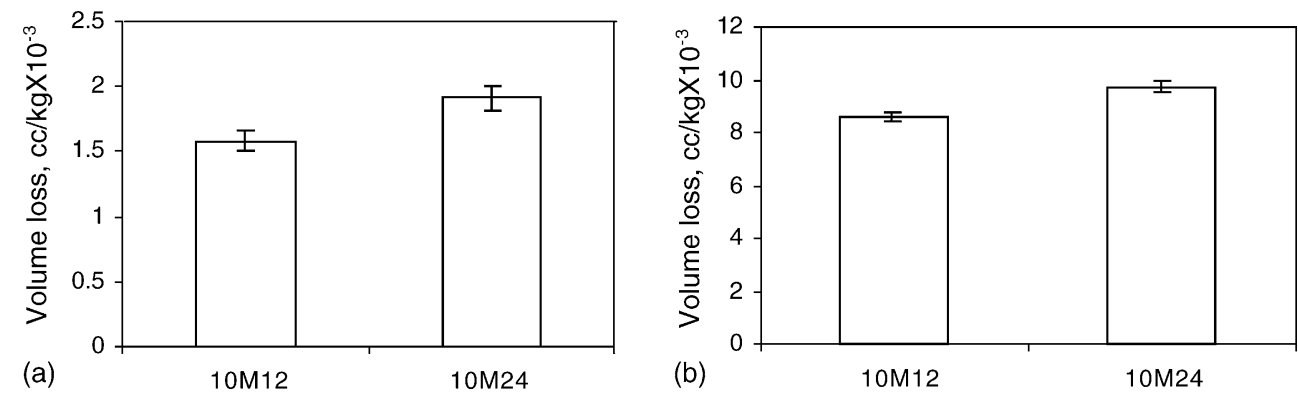

Fig. 9. (a) $10 \%$ metal cooled as-cast samples of different section sizes showing response to abrasion (b) $10 \%$ metal cooled as-cast samples of different section sizes showing response to erosion. 

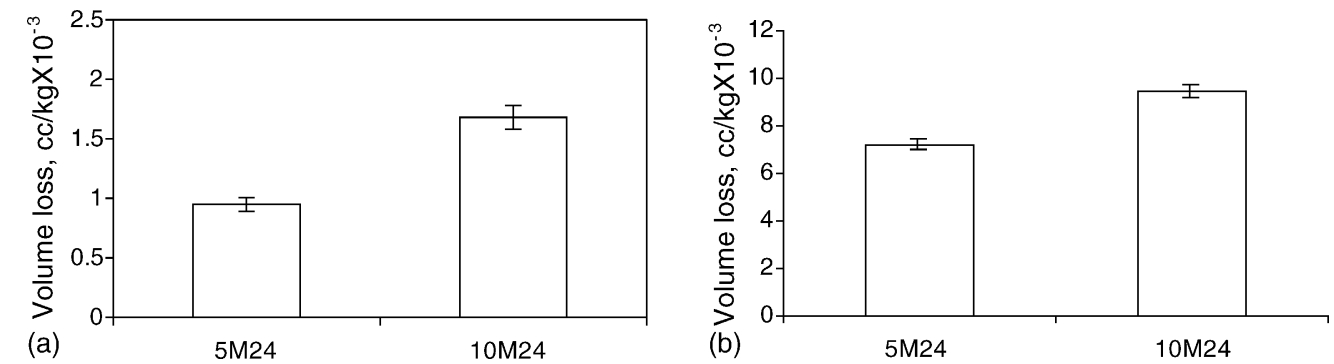

Fig. 12. (a) Loss due to abrasion in 5 and $10 \%$ manganese bearing heat treated samples; (b) loss due to erosion in 5 and $10 \%$ Manganese bearing heat treated samples.

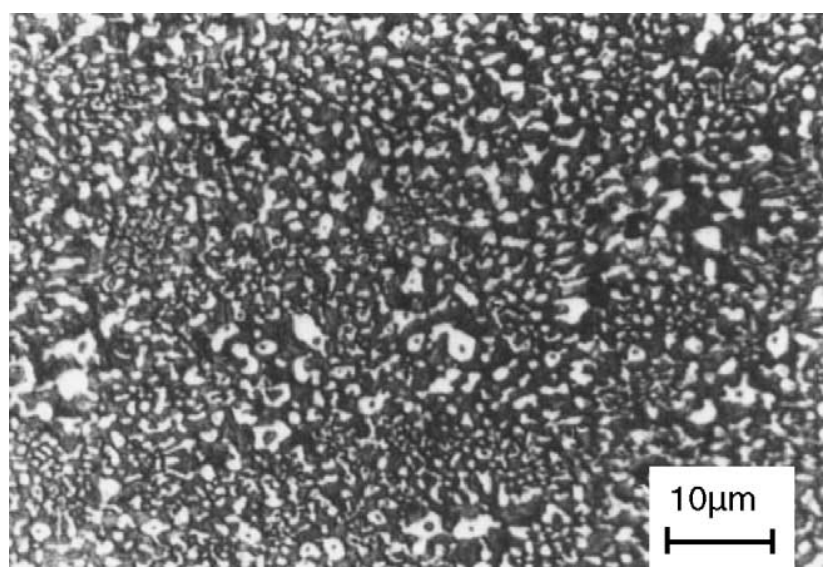

Fig. 13. Optical micrograph of heat treated 5M12 sample.

juncture, it is worth recalling a similar exercise was carried out by Gundlach and Parks [16] for studying the effect of cooling rate through varied section sizes on the abrasion resistance property of high chromium iron. In that work, the high stress grinding abrasion resistance decreases with increase in section size from 16 through 32 through 50 to $64 \mathrm{~mm}$ in sand mould cast samples. Thus, the data trends in the present study on the effect of section size on the wear properties is in good agreement with those reported $[15,16]$ on ferrous-based systems.

The effect of heat treatment is now considered for the samples having different manganese content and varied section thickness. The optical micrographs of the heat treated samples 5M24 and 10M24 are shown in Figs. 10 and 11, respec-

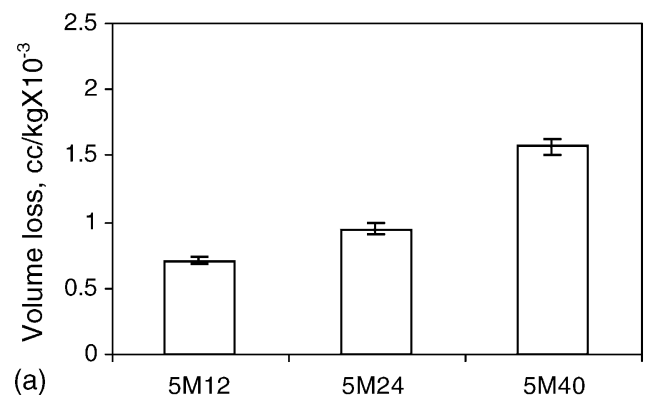

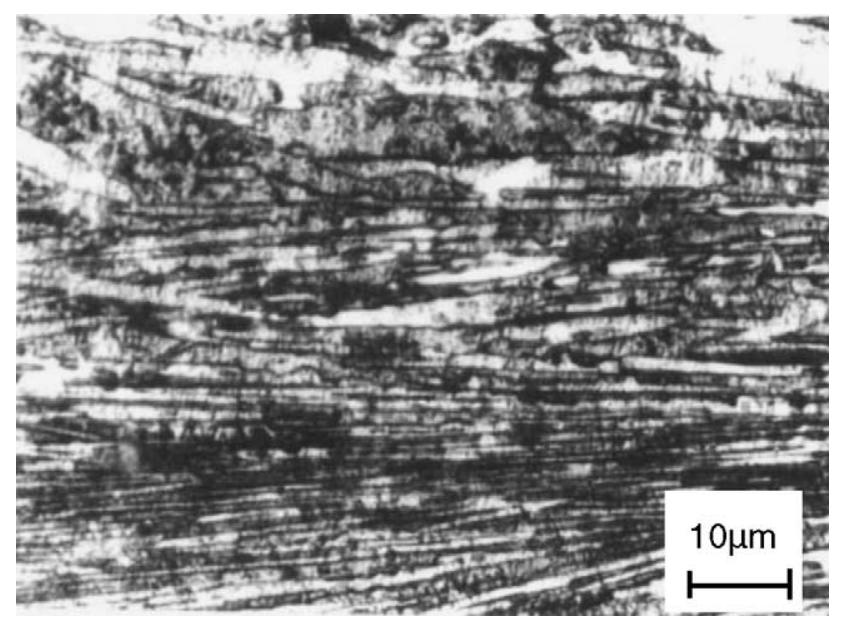

Fig. 14. Optical micrograph of heat treated 5M40 sample.

tively. The data on abrasion and erosion for these samples from the viewpoint of heat treatments are shown in Fig. 12a and $b$, respectively. The heat treated sample 5M24 shows fine (compare Fig. 10 with Fig. 2) and some hexagonal carbides (marked as 'A', Fig. 10) with lower amount (50\%; Table 2) of austenite (marked by arrows, Fig. 10). On the other hand, the sample 10M24 upon heat treatment shows coarser carbides (Fig. 11), which are either medium (marked as ' $\mathrm{M}$ ') or long (marked as 'L' i.e., rod-like) in sizes with a moderate increase in hardness (from 50 to $53 \mathrm{HRC}$; Table 2) and decrease in RA (from 63 to $60 \%$ ). Thus, the microstructural modifications in 5M24 are responsible for higher hardness (Table 2) and lower wear losses (Fig. 12a and b).

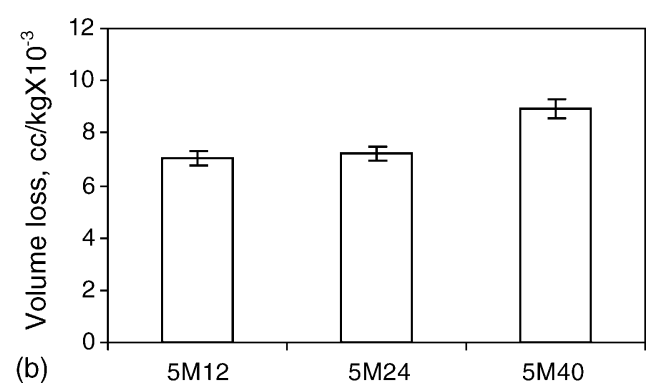

Fig. 15. (a) $5 \%$ metal cooled heat treated samples of different section sizes showing response to abrasion; (b) $5 \%$ metal cooled heat treated samples of different section sizes showing response to erosion. 


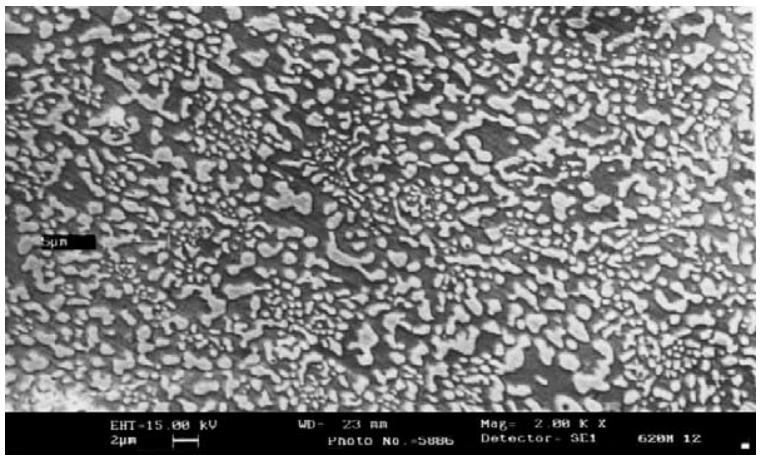

Fig. 16. Scanning electron micrograph of heat treated 5M12 sample.

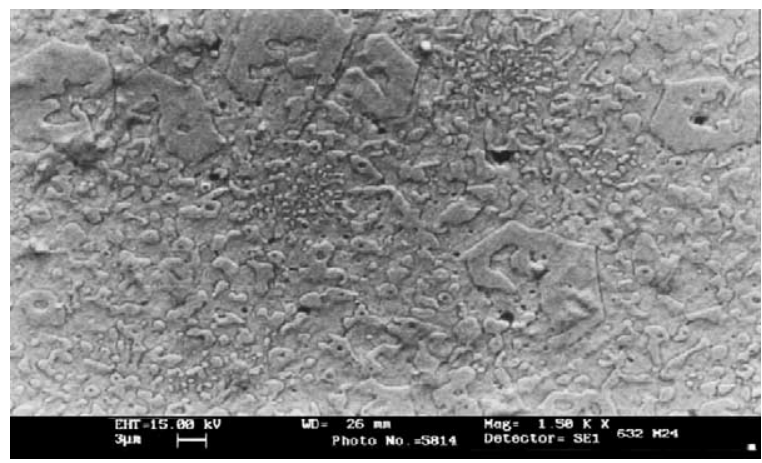

Fig. 17. Scanning electron micrograph of heat treated 5M24 sample.

Now, coming to the effect of heat treatment due to change in the casting section size, the microstructural features of 5M12 and 5M40 are shown in Figs. 13 and 14, respectively. For the sake of interpretation of results due to the section size variation in 5\% manganese bearing case, Fig. 10 presented earlier in respect of 5M24 sample is again considered here. The data on abrasion and erosion for these samples from the viewpoint of heat treatment are shown in Fig. 15a and $b$, respectively. The SEM photographs of deep etched 5\% manganese bearing samples (i.e., 5M12 and 5M24) are shown in Figs. 16 and 17, respectively to support the optical micrograph features. The examination of the optical microstructures reveal very fine globular type of carbides due to spherodization $[17,18]$ in 5M12 (Fig. 13), fine plus some hexagonal carbides in 5M24 (Fig. 10) and non-globular (rodlike type) and less randomly distributed carbides in 5M40

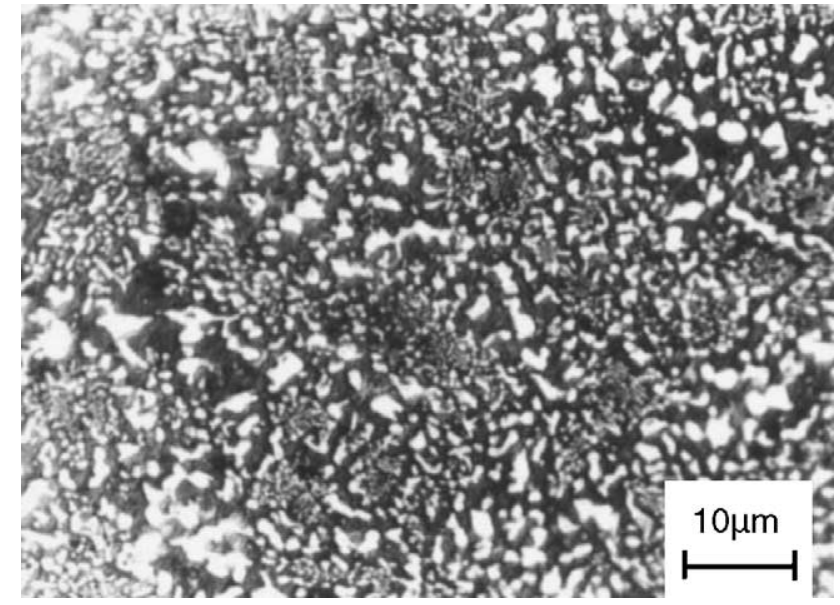

Fig. 18. Optical micrograph of heat treated 10M12 sample.

(Fig. 14), respectively. The austenite level (Table 2) also increases with an increase in the section size. The sample 5M12 having higher hardness (63 HRC; Table 2) displays very fine carbides dispersed favourably (Fig. 13) with a higher CV (29\%) and lower austenite $(47 \%)$ retention. As regards the SEM photograph of 5M12 (Fig. 16) is concerned, it contains very fine carbide particles with the average particle size less than 2 microns, thus corroborating the optical micrograph (Fig. 13) deduction (on spherodization). The sample 5M24, on the other hand, shows medium-sized and some hexagonal primary carbides (Fig. 17), which are much larger, compared to 5M12 (Fig. 16). Consequently, this sample (i.e., 5M12) exhibits the lowest wear losses. On the other hand, 5M40 shows lower hardness (56 HRC) due to the presence of less randomly distributed carbides of larger (rod-like) sizes (Fig. 14) resulting in higher wear losses (Fig. 15a and b).

As regards the optical micrographs of the higher manganese (i.e., 10M12 and 10M24) bearing heat-treated samples due to the change in the section size, these are shown in Fig. 18 and Fig. 11, respectively. The corresponding abrasion and erosion data are shown in Fig. 19a and b, respectively. The optical micrograph of 10M12 (Fig. 18) reveals fine carbides distributed uniformly in an austenitic matrix $(55 \%$; Table 2) with higher hardness (55 HRC) and better wear resistance, where as 10M24 shows medium or long-sized coarse carbides (Fig. 11). Fig. 20 shows SEM photograph of deep
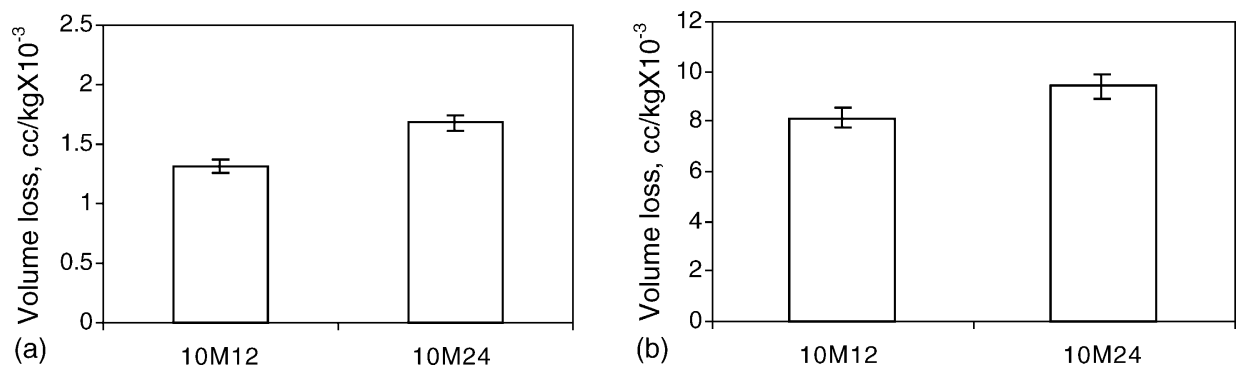

Fig. 19. (a) $10 \%$ metal cooled heat treated samples of different section sizes showing response to abrasion (b) $10 \%$ metal cooled heat treated samples of different section sizes showing response to erosion. 


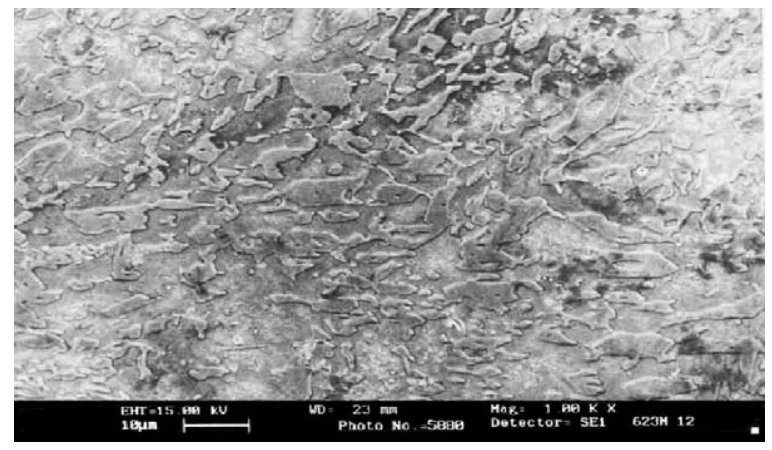

Fig. 20. Scanning electron micrograph of heat treated 10M12 sample.

etched 10M12 sample. Further, it is seen from the SEM photographs of $10 \mathrm{M} 12$ in comparison with 5M12, the latter features very fine globular carbides (Fig. 16), thus supporting the spherodization $[17,18]$ phenomena, where as the former shows carbides which are much larger in size (Fig. 20). Consequently, this 5M12 sample exhibits higher hardness and better wear properties. Thus, it is summarized that the heat treatment effect and the resulting higher wear resistance is best shown in the least section size casting irrespective of the manganese concentration employed in this work.

\section{Conclusions}

- On the basis of wear resistance and hardness, adding $5 \mathrm{wt} . \%$ manganese to iron chromium alloys is better than adding $10 \mathrm{wt} . \%$ manganese.

- Higher hardness and better wear resistance are seen for the sample having higher carbide volume in the matrix.

- Least section size as-cast metal cooled samples featuring fine globular type of carbides show higher hardness and superior wear resistance compared to the higher section sizes. For given manganese level, the wear resistance is better for smallest section size sample.

- The spherodization of carbides due to heat treatment contributes to higher hardness and better wear resistance over the as-cast samples for the two conditions attempted in this work.

\section{Acknowledgements}

The keen encouragement given by the Chairman, Department of Metallurgy, Indian Institute of Science to carry out this work is gratefully acknowledged. The authors also wish to thank the Director General and the management of Central Power Research Institute for according permission to publish the paper. The help rendered by Mr. D. Mollaiah and
A.V. Narayan of IISc., Mr. R.K. Kumar and Mr. P. Thomas of CPRI in preparing the manuscript and conducting the experiments at CPRI is acknowledged. The assistance extended by Sri. T.R. Raghu Kiran, Sri. Amit Kiran and Sri. Santosh V. Angadi of IISc and Sri. J. Balakrishna Sake of CPRI in preparing the text is acknowledged.

\section{References}

[1] P.R. Krishnamoorthy, S. Seetharamu, P. Sampathkumaran, Wear and erosion: basic concepts, in: Proceedings of the National Workshop on Wear and Erosion of Materials in Thermal Power Stations, CPRI, Bangalore, December, 1989, pp. A1-A19.

[2] I.M. Hutchings, A Text Book on, Tribology: Friction and Wear of Engineering Materials, Edward Arnold, A Division of Hodder Headline Group, London, 1992, pp. 77-82 and 133-134.

[3] T. Srinivasan, A.K. Patwardhan, M.L. Mehta, Effect of manganese and copper additions on microstructure of white cast irons, ASM Int. Cast Metals J. (1977) 57-60.

[4] P. Yan, Q. Zhou, The influence of boron on the abrasion wear resistance of $17 \% \mathrm{cr}$ white iron, in: International Conference on Wear of Materials ASME, New York, 1987, pp. 743-752.

[5] B.M. Hebbar, S. Seshan, Fracture toughness of high chromium irons, AFS Trans. 102 (1994) 349-356.

[6] F. Maratray, Improvement of and research into abrasion resistant materials, AFS Int. Cast Metals J. (1981) 55-61.

[7] S. Seetharamu, P. Sampathkumaran, R.K. Kumar, Erosion resistance of permanent moulded high chromium iron, Wear 186/187 (1995) 159-167.

[8] P. Sampathkumaran, R.K. Kumar, S. Seetharamu, Development of wear resistant high chromium, manganese and titanium alloyed irons, CPRI technical report no. 381 (2001) 43-50.

[9] S. Seetharamu, P. Sampathkumaran, P.R. Krishnamoorthy, Wear and erosion in thermal power station components, CPRI technical report no. 219 (1991) 11-25.

[10] A.W. Ruff, L.K. Ives, Measurement of solid particle velocity in erosion wear, Wear 35 (1975) 195-199.

[11] Annual Book of ASTM Standards: G 65-94, Section 3, 03-02 ASTM, Philadelphia, 1984, pp. 239-250.

[12] G. Laird, Some comments on white cast iron microstructure and wear properties, AFS Trans. 128 (1993) 497-504.

[13] A. Basak, J. Penning, J. Dellewyns, Effect of manganese on wear resistance and impact strength of $12 \%$ chromium white iron, AFS Int. Cast Metals J. (1981) 12-17.

[14] Karl Heinz Zum Gahr, D.V. Doane, Optimizing fracture toughness and abrasion rsistance in white cast irons, Metallurg. Trans. 11A (1980) 612-620.

[15] S. Junyl, J. Yuding, The effect of orientation and thickness of carbides on abrasive wear resistance of high chromium iron, in: International Conference on Wear of Materials, ASME, New York, 1987, pp. 661-671.

[16] R.B. Gundlach, J.L. Parks, Influence of abrasive hardness on wear resistance of high chromium irons, in: International Conference on Wear of Materials, ASME, New York, 1977, pp. 126-131.

[17] T.E. Norman, A. Soloman, V. Douglas, Doane, martensitic white irons for abrasion resistant castings, AFS Trans. 77 (1977) 242-256.

[18] C.P. Tabrett, I.R. Sare, M.R. Ghomashchi, Microstructure-property relations in high chromium white iron alloys, Int. Mater. Rev. 41 (1996) 59-82. 